\title{
Diversidad, densidad poblacional y distribución espacial de Columnea (Gesneriaceae) en la Reserva Natural Río Ñambí, Nariño, Colombia
}

\author{
Oscar Humberto Marín-Gómez* , Marisol Amaya-Márquez \\ Instituto de Ciencias Naturales, Universidad Nacional de Colombia, Bogotá, D.C., Colombia
}

\begin{abstract}
Resumen
Se estudió la diversidad, la densidad poblacional y la distribución espacial y vertical de las especies de Columnea (Gesneriaceae) en la Reserva Natural Río Ñambí en el suroccidente de Colombia. Se ubicaron 100 cuadrantes (10 x $10 \mathrm{~m}$ ) con el fin de cuantificar la diversidad alfa, la densidad y los patrones de distribución vertical y espacial de las especies de Columnea. La diversidad y la densidad poblacional de cada especie se analizaron con relación a la elevación. Se encontraron 15 especies de Columnea entre los 1.100 y los 1.500 msnm. La densidad de las especies varió con la elevación y fue mayor entre los 1.100 y los $1.300 \mathrm{msnm}$, con un patrón de distribución espacial agregado. La riqueza varió entre 10 y 12 especies por franja altitudinal, mientras que la diversidad alfa se redujo entre los 1.400 y los $1.500 \mathrm{msnm}$. La similitud en la composición de especies entre los sitios fue baja, lo que indica una gran diversidad beta para este ensamble. Los resultados de este estudio indican que la Reserva Natural Río Ñambí presenta la riqueza de Columnea más alta registrada para cualquier localidad hasta el momento. Se discuten las diferencias en la densidad y la distribución vertical de las especies y su relación con sus polinizadores y dispersores.
\end{abstract}

Palabras clave: Chocó biogeográfico, Columnea, densidad, diversidad, distribución espacial.

Diversity, population density and spatial distribution of Columnea (Gesneriaceae) in the Río Ñambí natural reserve, Nariño, Colombia

\begin{abstract}
We studied the diversity, population density and vertical and spatial distribution of the species of Columnea in the Río Ñambí natural reserve, southwestern Colombia. We divided the area under study in 100 plots $(10$ x 10 m) in order to quantify the alpha diversity, the density, and the vertical and spatial distribution pattern of Columnea species. The diversity and population density of each species were analyzed according to the elevation. Fifteen species of Columnea were recorded in altitudes ranging from 1100 to 1500 m.a.s.l. Species density varied with elevation and was highest between 1100 and 1300 m.a.s.l., showing a clumped spatial distribution pattern. Species richness varied between 10 and 12 species per altitudinal belt, while the alpha diversity decreased between 1400 and1500 m.a.s.l. The similarity of species composition was low among sites indicating a high beta diversity for this assemblage. The results of this study showed that the Río Ñambí natural reserve exhibits the highest Columnea richness recorded for a locality to date. We discuss here the differences in density and vertical distribution of Columnea species and their relationship with pollinators and dispersers.
\end{abstract}

Key words: Biogeographic Chocó, Columnea, density, diversity, spatial distribution.

\section{Introducción}

El Chocó biogeográfico constituye uno de los centros de endemismos de flora más importantes del planeta (Barthlott, et al., 2005), y en él predomina una gran diversidad y abundancia de epifitas vasculares (Gentry \& Dodson, 1987; Galeano, et al., 1998; Köster, et al., 2013), las cuales son elementos característicos de los bosques neotropicales (Gentry \& Dodson, 1987; Benavides, et al., 2006). Las epifitas usan a sus hospederos o forofitos únicamente como soporte mecánico y son importantes en la estructura y función de los ecosistemas debido a la captación y flujo de nutrientes, la regulación hídrica y el mantenimiento de la humedad del dosel (Benzing, 1990; Cavelier, et al., 1997), así como al suministro de recursos alimenticios y reproductivos para aves, anfibios y artrópodos (Nadkarni \& Matelson, 1989; Benzing, 1990; Stuntz, et al., 2002).

\footnotetext{
*Correspondencia:

Oscar Humberto Marín-Gómez, ohmaring@unal.edu.co

Recibido: 18 de noviembre de 2014

Aceptado: 13 de mayo de 2014
} 
Las epifitas vasculares constituyen entre el 10 y el $25 \%$ de la flora local en las franjas de bosque tropical lluvioso a premontano (Nieder, et al., 2001; Wester, et al., 2011), mientras que en los bosques montanos pueden alcanzar el 50 \% (Kelly, et al., 1994). Estas plantas están representadas por 27.614 especies, 913 géneros y 73 familias (Zotz, 2013), de las cuales las Orchidiaceae, Araceae, Bromeliaceae, Piperaceae, Gesneriaceae, Melastomataceae y Ericaceae son las más diversas (Gentry \& Dodson, 1987; Benavides, et al., 2006). Las gesneriáceas epifitas alcanzan hasta el $5 \%$ de la flora vascular en los bosques lluviosos tropicales de zonas bajas (Wester, et al., 2011), y junto con las Ericaceae y Bromeliaceae constituyen recursos importantes para las comunidades de colibríes, los cuales son sus principales polinizadores (Amaya-Márquez, et al., 2001; Gutiérrez, et al., 2004).

La familia Gesneriaceae tiene alrededor de 600 especies (20\%) y 20 géneros (16\%) con hábito epifito (Wiehler, 1983; Gentry \& Dodson, 1987; Zotz, 2013). Sus hábitos de crecimiento varían de epífito a hemiepifito; en este último la planta inicia su vida como terrestre, con un sistema radical anclado en el suelo, y crece trepada a un forofito. El sistema radical puede o no perder conexión con el suelo (hemiepifito primario), o puede germinar y desarrollarse en la corteza descendiendo al suelo (hemiepifito secundario), o hacerlo directamente en el forofito sin alcanzar el suelo (Benzing, 1990; Salinas, et al., 2010; Wiehler, 1983; Weber, 2004). Columnea es el género más diverso de las gesneriáceas neotropicales, con 205 especies (Möller \& Clark, 2013; Weber, et al., 2013). Son plantas de hábito terrestre (hierbas o sufrútices), trepador (enredadera-herbáceo o lianescenteleñoso) y estrictamente epifito, que varían en la disposición del tallo (erecto, extendido o péndulo), el arreglo de las hojas en cada nudo (anisofilas o isofilas), y la orientación de las inflorescencias, que pueden ser adpresas al vástago y erectas o exertas del vástago y erectas o péndulas, combinación de rasgos que incrementa su diversidad (Kvist \& Skog, 1993; Weber, 2004). El género Columnea L. se distingue de otros de la familia por tener frutos carnosos indehiscentes de color opaco, globosos a ovoides (Wiehler, 1983; Smith, 1994), flores tubulares, subventricosas o bilabiadas, de color rojo, púrpura, naranja o amarillo y, frecuentemente, disposición anisofila en las hojas de cada nudo (Weber, 2004). Además, varias especies presentan patrones distintivos de manchas de color rojo o púrpura en la parte abaxial de las hojas, las cuales constituyen una señal de atracción permanente para sus polinizadores (Stiles, 1981; Kvist \& Skog, 1993; Amaya-Márquez, 1996).

Las plantas del género Columnea tienden a presentar una distribución dispersa con pocos individuos por población (Kvist \& Skog, 1993), sin embargo, solo hay un estudio que ha evaluado su densidad poblacional. Amaya-Márquez (1999) llevó a cabo un estudio para determinar si la edad y el grado de perturbación del bosque afectaban la densidad de la población de siete especies de Columnea. Sus resultados indicaron que la diferencia de edad del bosque no afectaba los patrones de densidad de la mayoría de especies, excepto en Columnea medicinalis y Columnea rubriacuta, las cuales fueron más abundantes en las zonas de rastrojo. Las diferencias en la abundancia de estas dos especies se explicaron por: 1) su capacidad para aprovechar la disponibilidad de luz; 2) una mayor tolerancia para soportar cambios microclimáticos asociados con la perturbación; 3) el comportamiento de dispersores como las tangaras, que viajan en bandadas mixtas y dispersan sus frutos, así como el forrajeo en rutas de su polinizador, Phaethornis syrmatophorus, y 4) un sistema autocompatible que favorece la colonización (Amaya-Márquez, 1999).

A pesar de la gran diversidad exhibida por las gesneriaceas neotropicales, son escasos los estudios en los que se analizan los patrones de distribución y densidad poblacional (AmayaMárquez, 1999; Salinas, et al., 2010). Esta información es importante para entender sus requerimientos de hábitat y el estado poblacional, los efectos del impacto de los cambios antrópicos y los procesos de extinción y estado de conservación (Kvist, et al., 2004), así como sus interacciones con los polinizadores y el éxito reproductivo de las plantas (Feisinger, et al., 1991). En este trabajo se identificaron las especies de Columnea presentes en la Reserva Natural Río Ñambí, y se determinó su diversidad, densidad poblacional y distribución espacial y vertical.

\section{Área de estudio}

La Reserva Natural Río Ñambí se encuentra ubicada en el corregimiento de Altaquer, municipio de Barbacoas, departamento de Nariño, suroccidente de Colombia, a $1^{\circ} 18^{\prime} \mathrm{N}$ y $78^{\circ} 05^{\prime} \mathrm{O}$ (Figura 1). Tiene una extensión de 1.400 ha, con un gradiente altitudinal entre los 1.100 y los $1.900 \mathrm{~m}$, aunque el terreno solo es accesible en las zonas bajas (Salaman, 2001). Comprende zonas de vida de ecotono entre bosque pluvial tropical y pluvial premontano (Gutiérrez, et al., 2004). La precipitación anual promedio es de $7.160 \mathrm{~mm}$, con un patrón unimodal de lluvias con picos en mayo y octubre (Salaman, 2001). Los datos recolectados en la estación de Junín, a 7 km de Ñambí, indican que la precipitación anual promedio es de $8.825 \mathrm{~mm}$ (Franco-Rosselli, et al., 1997). La temperatura anual promedio es de $19{ }^{\circ} \mathrm{C}$, con una variación promedio en el día de $5,5^{\circ} \mathrm{C}$; la humedad relativa es de $100 \%$ debido a la saturación constante de neblina que reduce drásticamente la intensidad de la luz solar (Salaman, 2001). En Ñambí son frecuentes las tormentas fuertes a lo largo del año, que causan un impacto importante en la estructura del bosque al dejar claros de hasta $2.000 \mathrm{~m}^{2}$ (Salaman, 2001).

Ñambí se caracteriza por una cobertura representativa de bosque primario, aunque en algunas áreas está intervenido por la extracción selectiva de maderas como el barniz de Pasto (Elaeagia utilis) o de palmas para la construcción de senderos (Franco-Rosselli, et al., 1997). La vegetación se 


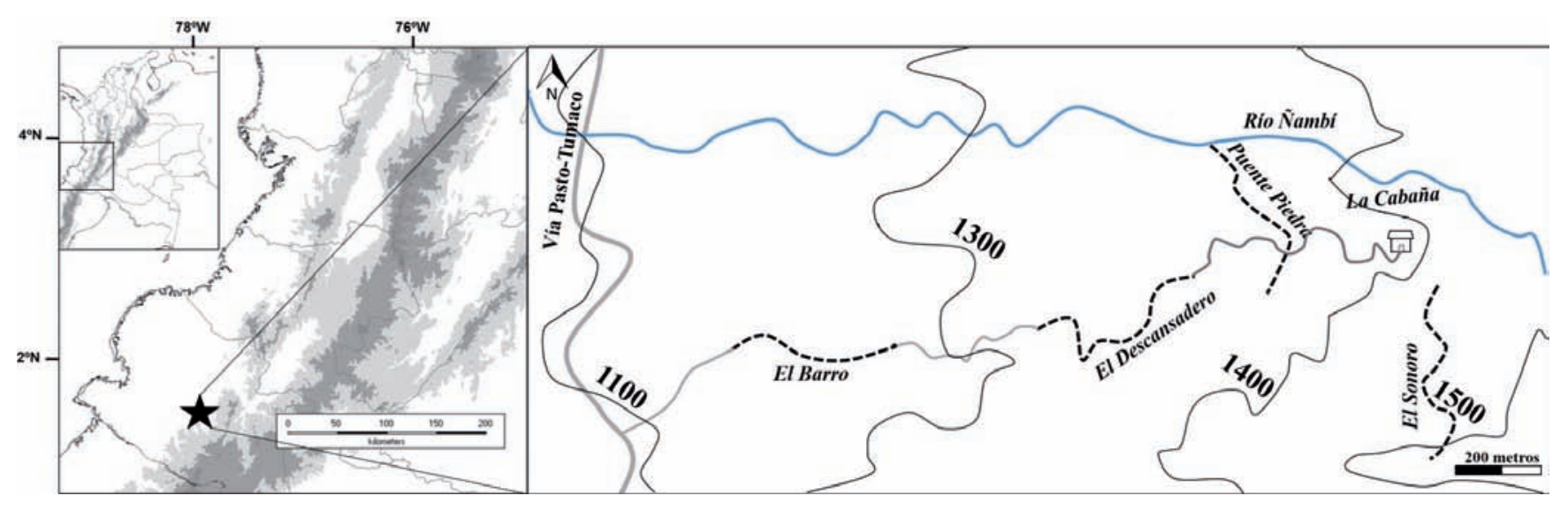

Figura 1. Ubicación de la Reserva Natural Río Ñambí con los cuatro sitios de muestreo entre los 1.100 y los 1.500 msnm.

caracteriza por un dosel de 25 a $30 \mathrm{~m}$, una gran densidad de epifitas (Araceae, Ericaceae, Bromeliaceae y Gesneriaceae), un sotobosque denso, y una dominancia de palmas como Welfia regia, Wettinia castanea y Wettinia kalbreyeri (Franco-Rosselli, et al., 1997). Los elementos florísticos con mayor diversidad en Nambí pertenecen a las familias Rubiaceae, Araceae y Melastomataceae (Franco-Rosselli, et al., 1997).

En Ñambí se encuentran áreas de bosque primario intervenido, bosque secundario, borde de bosque y claros, inmersas en una matriz de bosque maduro (Gutiérrez, et al., 2004). Para abarcar este mosaico de hábitats y el gradiente de altitudes, se establecieron las siguientes cuatro trochas de estudio entre los $1.100 \mathrm{y}$ los $1500 \mathrm{~m}$ (Figura 1).

El Barro: bosque secundario entre los 1.100 y los $1.200 \mathrm{~m}$, con un sotobosque poco denso dominado por especies de Maranthaceae y Heliconiaceae, y un dosel de bajo porte, entre 10 y 15 m, debido a la extracción de madera. En esta zona predominan las especies de Rubiaceae, Melastomataceae, Clusiaceae, Cecropiaceae, Euphorbiaceae, Solanaceae y Marcgraviaceae.

EI Descansadero: bosque maduro intervenido debido a la tala selectiva y situado entre los 1.200 y los $1.300 \mathrm{~m}$. El dosel alcanza hasta $30 \mathrm{~m}$ con especies de Protium sp., Sapium glandulosum, Dendropanax macrophyllum, E. utilis, Bombacopsis patinoi y Casearia cajambrensis. El sotobosque es abierto y existe un alto nivel de epifitas de estrato medio y dosel.

Puente-piedra: bosque maduro con dominancia de palmas W. castanea y W. kalbreyeri, entre los 1.300 y los 1.400 $\mathrm{m}$. Es una zona muy húmeda de sotobosque denso con dominancia de especies de Melastomataceae, Rubiaceae, Dryopteridaceae, Araceae y Solanaceae. El dosel es abierto debido a la presencia de palmas, alcanza hasta los $25 \mathrm{~m}$ con algunos árboles emergentes de Sapium glandulosum, B. patinoi y Protium sp. Hay una gran densidad de epifitas debido a la humedad, con predominio de especies de Bromeliaceae, Araceae, Ericaceae, Cyclantaceae, Orchidiaceae y Gesneriaceae.

Quebrada La Sonadora: bosque maduro a 2,5 km de la vía Pasto-Tumaco, entre los 1.400 y los $1.500 \mathrm{~m}$, dominado por las palmas $W$. regia y $W$. castanea. El dosel alcanza una altura de $20 \mathrm{~m}$ con pocos árboles como Quaribea foenigracea. Hay una gran densidad de epifitas en la parte media de los troncos de las palmas. El sotobosque es abierto y está caracterizado por dominancia de Ossaea macrophylla, Miconia spp., Psychotria spp., y Palicourea spp.

\section{Métodos}

Entre mayo y julio de 2013 se ubicaron cuatro sitios de muestreo desde la entrada de la reserva hasta la cabaña, siguiendo un gradiente altitudinal entre los 1.100 y los 1.500 msnm (Figura 1). En cada sitio se delimitó un trayecto de $500 \times 10$ m, marcado cada $10 \mathrm{~m}$ con un código único. Para cada trayecto se seleccionaron 25 códigos con una tabla de números aleatorios, en cada uno de los cuales se montó una parcela de 10 x $10 \mathrm{~m}$ (Elzinga, et al., 1998). En cada parcela se contó una sola vez el número de individuos de cada especie de Columnea y se registró el hábito y la altura de cada planta. Para cada espécimen recolectado se registró el hábitat, la elevación, la posición geográfica y su estado reproductivo. Se hizo una colección de referencia para apoyar la identificación de cada especie mediante comparación con especímenes de referencia en el Herbario Colombiano de la Universidad Nacional de Colombia (COL) con las claves de Kvist \& Skog (1993).

Se calculó la densidad de cada especie de Columnea (ind/ ha), la frecuencia por sitio y el patrón de distribución espacial con el índice de Morisita (Krebs, 1999). Se comparó la distribución vertical de las especies y la densidad poblacional entre sitios por especie con una prueba de Kruskal-Wallis, seguida de una prueba de comparaciones múltiples de Z (Zar, 1999). Se visualizó la distribución de las especies por cuadrante y por sitio con un gráfico de 
coocurrencias. Se calculó el índice de distribución espacial con la función 'dispindmorisita' de la librería 'vegan' del programa R (Oksanen, et al., 2013). Se calcularon los dos valores críticos (Mclu, Muni) para el índice de Morisita y el índice estandarizado de Morisita (Imst) con valores entre los rangos de -1 a +1 (con $95 \%$ de intervalo de confianza), donde un Imst $=0$ muestra una distribución aleatoria, un Imst $>0$, una distribución agregada, y un Imst $<0$, una distribución uniforme (Krebs, 1999). Por último, se calcularon los índices de diversidad alfa de Shannon-Wiener y Simpson, y de diversidad beta con el índice de Bray Curtis (Krebs, 1999). Se comparó la variación de la diversidad con respecto a la elevación con una prueba de Kruskal-Wallis, seguida por una prueba de comparaciones múltiples de Z (Zar, 1999).

\section{Resultados}

Se registraron 15 especies del género Columnea (Tabla 1; Figura 2); la distribución vertical varió significativamente entre especies $\left(H_{14,431}=217,5 ; p<0,0001\right.$; Figura 3). Se identificó a $C$. minutiflora, $C$. fimbricalyx, C. ceticeps y $C$. byrsina como epifitas restringidas al dosel, a excepción de C. minor, que se encontró entre el sotobosque $(2 \mathrm{~m})$ y el dosel; C. medicinalis y C. longinervosa se encontraron como hemiepifitas secundarias entre el sotobosque y el estrato medio (Figura 3). Las especies restantes se encontraron entre los 0 y 4 m, y entre ellas Columnea sp. nov. 1, C. eburnea,
C. picta y C. rubriacuta se encontraron predominantemente como hemiepifitas secundarias terrestres (Figura 3). La densidad de Columnea en la reserva fue de $203 \mathrm{ind} / \mathrm{ha}$. La densidad de las especies varió con relación a la elevación $\left(\mathrm{H}_{3}\right.$ ${ }_{100}=9,89 ; \mathrm{p}=0,02$ ) y fue mayor entre los 1.100 y los $1.300 \mathrm{~m}$ : C. albovinosa y Columnea sp. nov. 2 presentaron una mayor densidad entre los 1.100 y los $1.200 \mathrm{~m}$, C. rubriacuta entre los 1.300 y los $1.400 \mathrm{~m}$, y C. longinervosa entre los 1.400 y los $1.500 \mathrm{~m}$ (Tabla 1$)$.

La riqueza varió entre 10 y 12 especies por sitio y la diversidad varió con la elevación (Shannon-Wiener: $\mathrm{H}_{3}$, ${ }_{100}=27,7$; $\mathrm{p}=0,0001$; Simpson: $\mathrm{H}_{3,100}=32,6$; $\left.\mathrm{p}=0,0001\right)$. Se encontró una mayor diversidad entre los 1.100 y los $1.200 \mathrm{~m}$, con una disminución entre los 1.300 y los 1.400 m (Figura 4). El índice de Simpson mostró un patrón similar, pero con mayores valores entre los 1.400 y los $1.500 \mathrm{~m}$ (Figura 4). Las especies con mayor densidad, como C. albovinosa, $C$. rubriacuta, C. medicinalis, C. longinervosa, C. sp nov. 2 y C. filifera, se encontraron a lo largo del gradiente altitudinal, pero su densidad tendió a ser más alta en un sitio, presentando un patrón de distribución espacial agregado (Tabla 1; Figura 5). Ocho especies presentaron una frecuencia en los sitios de muestreo de entre 11 y $22 \%$ (Columnea sp. nov. 2, C. albovinosa, C. longinervosa, C. medicinalis, C. gigantifolia, C. rubriacuta, C. minor y C. filifera), en tanto que las especies restantes presentaron una frecuencia baja, entre 1 y $6 \%$ (Figura 5 ).

Tabla 1. La densidad poblacional (ind/0,25ha) de las especies del género Columnea de la Reserva Natural Río Ñambí varía con la elevación y las especies más abundantes presentan una distribución espacial agregada (Imst: índice de Morisita).

\begin{tabular}{|c|c|c|c|c|c|c|c|}
\hline \multirow{2}{*}{ Especie } & \multicolumn{4}{|c|}{ Elevación (m) } & \multirow{2}{*}{$\begin{array}{l}\text { Densidad } \\
\text { (ind/1ha) }\end{array}$} & \multirow{2}{*}{$\begin{array}{c}\text { Kruskal-Wallis } \\
\qquad\left(\mathbf{H}_{3,100}\right)\end{array}$} & \multirow{2}{*}{ Imst } \\
\hline & $1100-1200$ & $1200-1300$ & $1300-1400$ & $1400-1500$ & & & \\
\hline C. albovinosa (M. Freiberg) J.L. Clark \& L.E. Skog & 16 & 14 & 1 & & 31 & $23,2 * *$ & 0,51* \\
\hline C. rubriacuta (Wiehler) L.P. Kvist \& L.E. Skog & & 3 & 15 & 9 & 27 & $12,4 * * *$ & $0,52 *$ \\
\hline C. medicinalis (Wiehler) L.E. Skog \& L.P. Kvist & 10 & 7 & 7 & 2 & 26 & $4,3^{\mathrm{NS}}$ & 0,51* \\
\hline C. longinervosa L.P. Kvist \& L.E. Skog & 1 & 2 & 7 & 14 & 24 & $17,5 * *$ & $0,32 *$ \\
\hline Columnea sp. nov. 2 & 17 & 3 & 1 & 1 & 22 & $28,7 * * *$ & $0,51 *$ \\
\hline C. filifera (Wiehler) L.E. Skog \& L.P. Kvist & 5 & 10 & 6 & & 21 & $5,2^{\mathrm{NS}}$ & $0,54 *$ \\
\hline C. gigantifolia L.P. Kvist \& L.E. Skog & 8 & 4 & 2 & 4 & 18 & $4,1^{\mathrm{NS}}$ & $0,09 *$ \\
\hline C. minor (Hook.) Hanst & 4 & 6 & 5 & 1 & 16 & 4,6 Ns & $0,17 *$ \\
\hline Columnea sp. nov. 1 & & 3 & & 3 & 6 & 6,3 NS & $-0,1^{*}$ \\
\hline C. byrsina (Wiehler) L.P. Kvist \& L.E. Skog & 3 & & 1 & 1 & 5 & 4,0 NS & $-0,08^{*}$ \\
\hline C. minutiflora L.P. Kvist \& L.E. Skog & 1 & & 1 & & 2 & $2,1^{\mathrm{NS}}$ & $-0,01 *$ \\
\hline C. ceticeps J.L. Clark \& J.F. Smith & & 1 & & & 1 & & \\
\hline C. eburnea (Wiehler) L.P. Kvist \& L.E. Skog & 1 & & & & 1 & & \\
\hline C. fimbricalyx L.P. Kvist \& L.E. Skog & & & & 1 & 1 & & \\
\hline C. picta H. Karst. & 1 & & & & 1 & & \\
\hline Total & 68 & 53 & 46 & 36 & 203 & & \\
\hline
\end{tabular}

$* \mathrm{p} \leq 0,05 ; * * \mathrm{p} \leq 0,01 ; * * * \mathrm{p} \leq 0,001 ;{ }^{\text {NS }}$ No significativo 


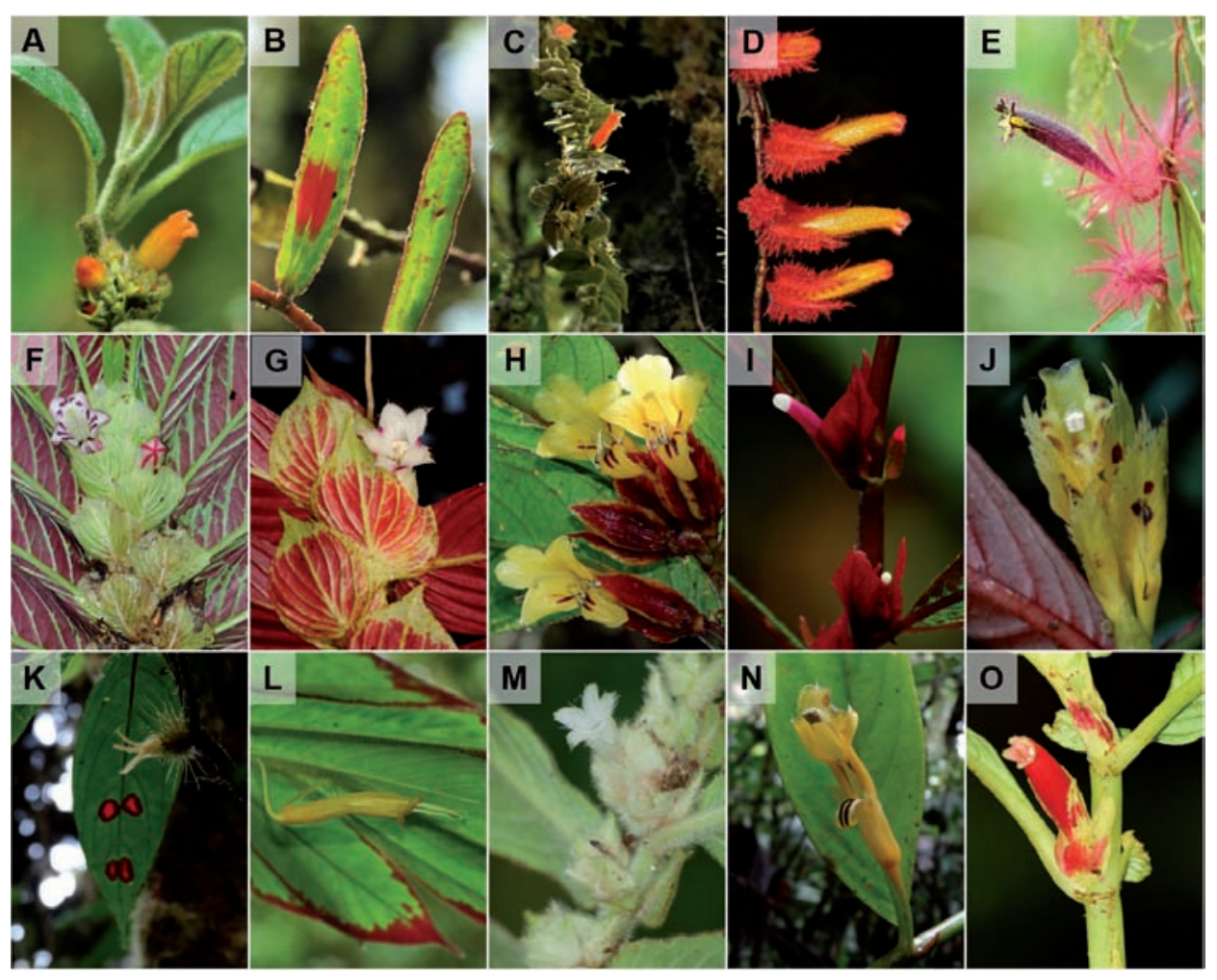

Figura 2. Fotografías de las especies de Columnea presentes en la Reserva Natural Río Nambí. A: C. minutiflora; B: C. byrsina; C: C. ceticeps; D: C. fimbricalyx; E: C. minor; F: C. medicinalis; G: C. longinervosa; H: C. gigantifolia; I: C. albovinosa; J: Columnea sp. nov 2; K: C. filifera; L: Columnea sp. nov 1; M: C. eburnea; N: C. picta; O: C. rubriacuta.

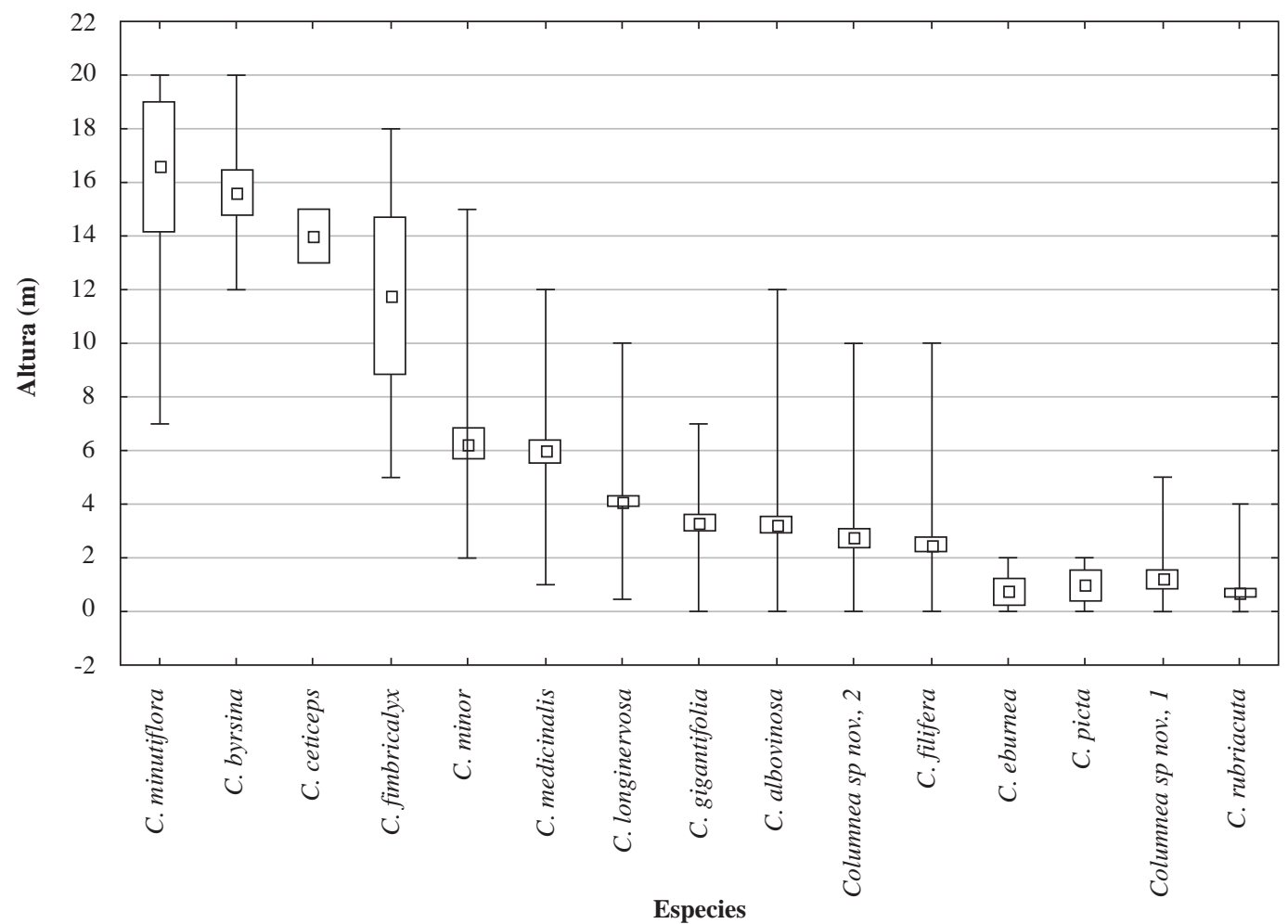

Figura 3. Segregación vertical de las especies de Columnea en la Reserva Natural Río Ñambí desde el suelo hasta el dosel. Se presenta el valor promedio, el error estándar (cajas) y el rango. 

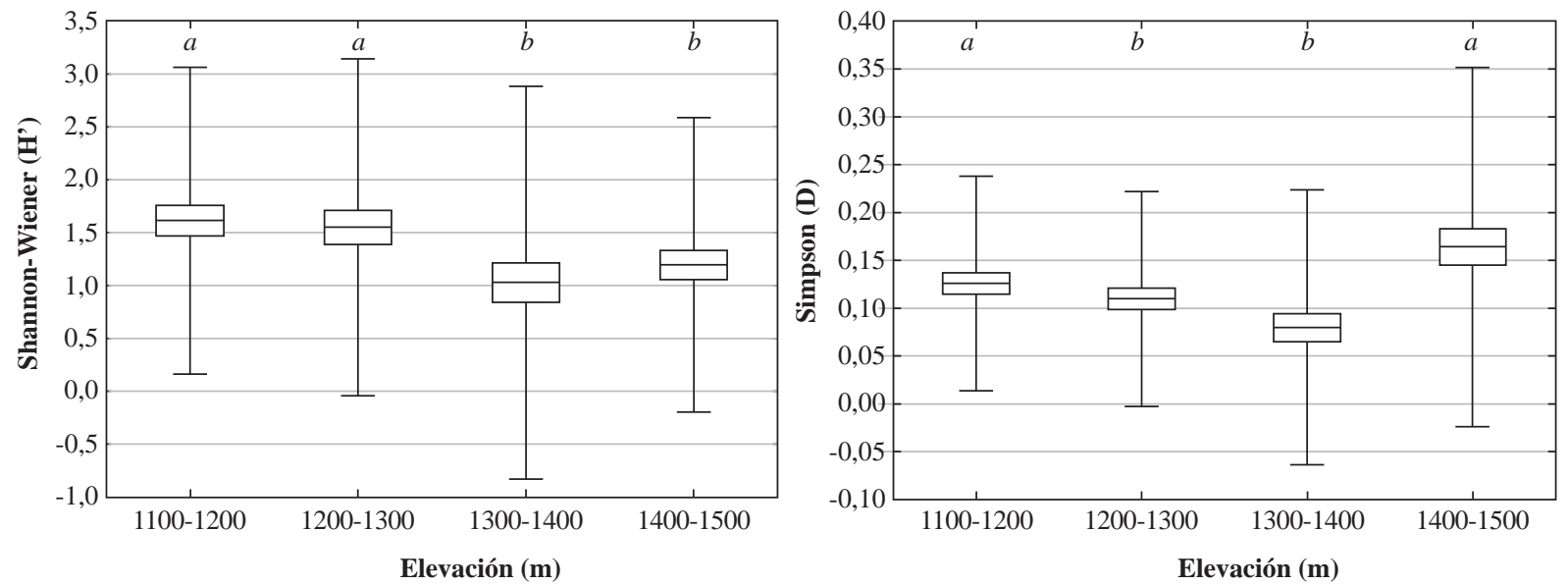

Figura 4. Los índices de diversidad de Columnea en la Reserva Natural Río Ñambí fueron bajos, la mayor diversidad ocurrió en las zonas bajas (Shannon-Wiener), mientras que entre los 1.400 y los $1.500 \mathrm{~m}$ hubo mayor dominancia (Simpson). Las letras iguales indican grupos homogéneos según la prueba de comparaciones múltiples.

1100-1200m
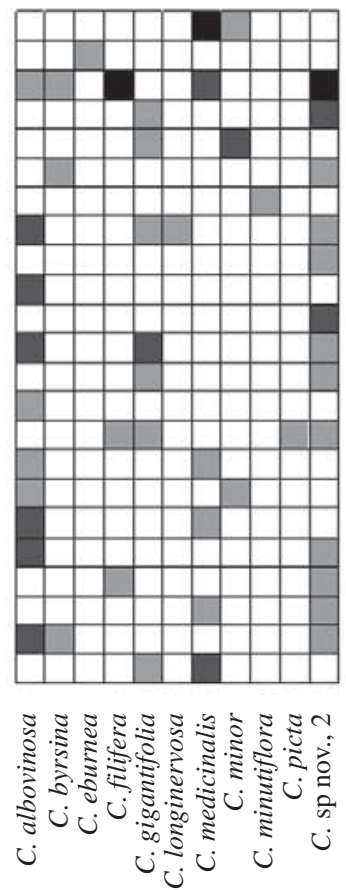

1200-1300m
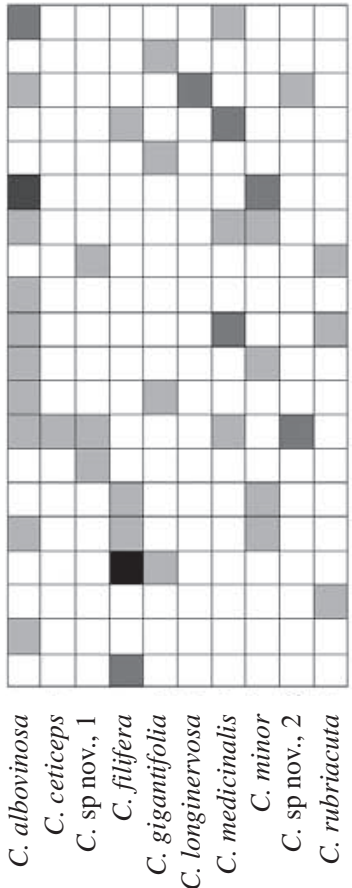

1300-1400m
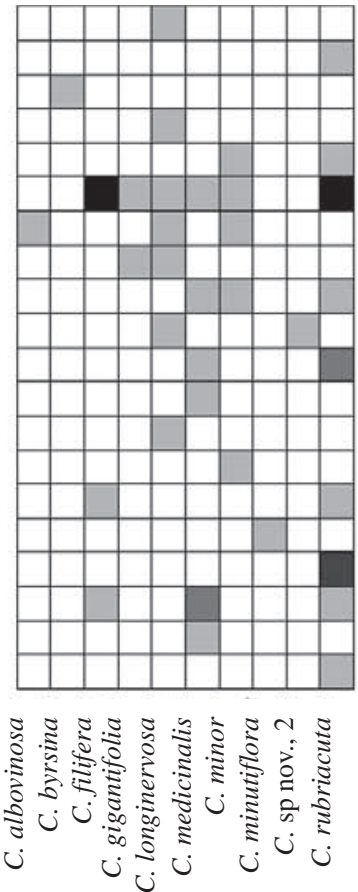

1400-1500m
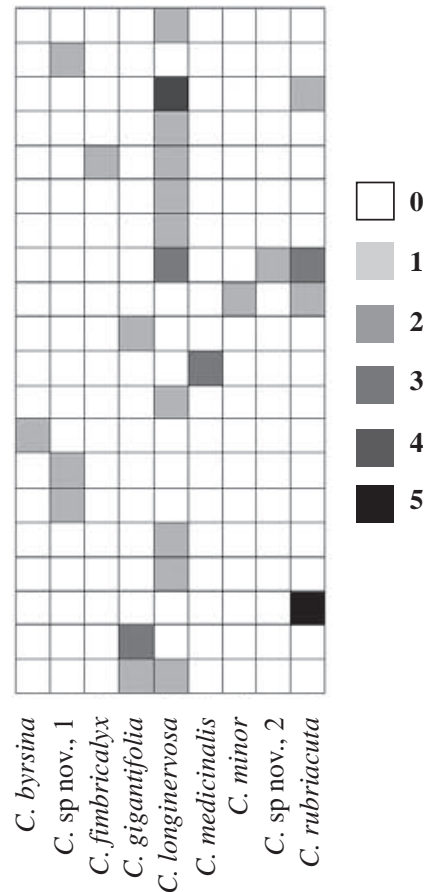

Figura 5. El gráfico de coocurrencia de las especies de Columnea por cuadrante y por sitio indica una variación en la frecuencia, la densidad, la distribución espacial y la dominancia de la Reserva Natural Río Nambí.

La similitud en la composición de especies entre los sitios fue baja, con un $38 \%$. Con el índice de Bray Curtis se dividieron los sitios en dos grupos: 1) entre los 1.100 y los $1.300 \mathrm{~m}$, con un $60 \%$ de similitud, y 2) entre los $1.300 \mathrm{y}$ los $1.500 \mathrm{~m}$, con un $54 \%$, lo que indica una gran diversidad beta para este ensamble. Además, cuatro especies fueron exclusivas de cada franja: C. eburnea y C. picta $(1.100 \mathrm{y}$ $1.200 \mathrm{~m})$, C. ceticeps $(1.200$ y $1.300 \mathrm{~m})$ y C. fimbricalyx (1.400 y $1.500 \mathrm{~m})$.

\section{Discusión}

Todas las especies de Columnea registradas en este estudio se encuentran restringidas a la vertiente del Pacífico de los Andes occidentales de Ecuador y Colombia (Kvist \& Skog, 1993). La única especie que alcanza la vertiente occidental de la Cordillera Central es C. ceticeps, en las inmediaciones del departamento de Antioquia (Smith, et al., 2013). C. rubriacuta, C. minor y C. picta son especies 
de amplia distribución y son comunes entre los 0 y los 2.000 msnm, mientras que C. albovinosa y $C$. fimbricalyx se han registrado en una franja angosta entre los 400 y los 600 m (Kvist \& Skog, 1993). Nuestros resultados amplían la distribución altitudinal de cuatro especies: $C$. ceticeps, que se ha registrado entre los 1.900 y los $2.900 \mathrm{~m}$ (Smith, et al., 2013), en Ñambí se encontró a los 1.400 m, aunque fue una especie rara (1 ind/ha). C. eburnea, que se ha registrado entre los 0 y los 850 m (Kvist \& Skog, 1993), en este estudio se encontró a los $1.100 \mathrm{~m}$. C. filifera es común entre los 680 y los 1.300 m (Kvist \& Skog, 1993), pero pudo encontrarse hasta los $1.500 \mathrm{~m}$ según la evidencia de este estudio. $C$. fimbricalyx habita entre los 700 y los 1.100 m (Kvist \& Skog, 1993), pero en Ñambí se la encontró habitando entre los 1.400 y los $1.600 \mathrm{~m}$. También se destaca el hallazgo de dos nuevas especies con distribución restringida al Chocó biogeográfico.

En la Reserva Natural Río Nambí se encuentran alrededor de 38 especies de gesneriáceas (Marín-Gómez, observación personal), con dominancia del género Columnea, el cual es abundante en toda la reserva. La gran riqueza de Columnea en Ñambí contrasta con otras localidades adyacentes en los Andes occidentales de Colombia y Ecuador, en donde se han registrado entre ocho y 13 especies por localidad (Dodson \& Gentry, 1978; Jaramillo \& Jorgesen, 1989; Silverstone-Sopkin \& Ramos-Pérez, 1995; Amaya-Márquez, 1999; Jaramillo, 2001; Mendoza-Cifuentes \& Ramírez-Padilla, 2001; Gonzáles \& Jarvis, 2002; Rangel, 2004; Clark, et al., 2006). Estas diferencias en riqueza se pueden atribuir a que Ñambí es un ecotono entre vegetación de bosque pluvial de zonas bajas y bosque pluvial montano (Salaman, 2001), franja en la que se concentra la mayor diversidad de epifitas en el Neotrópico (Gentry \& Dodson, 1987; Kvist \& Skog, 1993). Además, los altos niveles de precipitación y la niebla constante, tal como ocurren en Ñambí, son factores que se han asociado con una mayor diversidad de epifitas (Gentry \& Dodson, 1987; Benzing, 1990) como Columnea (Kvist \& Skog, 1993).

Por otra parte, los valores de diversidad fueron muy bajos debido a que el ensamble está conformado por pocas especies y la mayoría están restringidas a unos pocos cuadrantes (Figura 5). Además, la distribución de las abundancias fue equitativa, como lo reflejan los valores de Simpson, con los cuales se estableció que solo entre los 1.400 y los $1.500 \mathrm{~m}$ había una marcada dominancia (por ejemplo de $C$. longinervosa; Figura 5). También se destacan los valores relativamente altos de diversidad beta debidos al recambio de especies que ocurre a los $1.300 \mathrm{y}$ a los $1.500 \mathrm{~m}$, el cual puede estar asociado con la disminución de la densidad a medida que se incrementa la elevación (Tabla 1), un patrón predominante de la flora del Chocó biogeográfico (Gentry \& Dodson, 1987; Galeano, et al., 1998; Rangel, 2004; Köster, et al., 2013).
La gran densidad y la frecuencia de las especies de Columnea indican que este género de plantas es un componente importante de la flora en Nambí, al igual que lo encontrado para la reserva La Planada (Amaya-Márquez, 1999). Además, los valores de densidad fueron similares a los encontrados para otras especies de Columnea: entre 4 y 26 ind/ha (Amaya-Márquez, 1999; Benavides, et al., 2006; Zotz \& Schultz, 2008). Las seis especies más abundantes (Tabla 1) presentaron un patrón de distribución agregado, el cual es típico de varios grupos de epifitas vasculares neotropicales (Gentry \& Dodson, 1987; Zotz, et al., 1999; Nieder, et al., 2000; Areválo \& Betancur, 2006). Este patrón de distribución ha sido relacionado con las características estructurales de los forofitos (Areválo \& Betancur, 2006), la disponibilidad de sustratos (Nieder, et al., 2000), la humedad y la intensidad lumínica (Areválo \& Betancur, 2006) y las restricciones de dispersión a larga distancia (Zotz, et al., 1999).

En el caso de Ñambí, las especies de Columnea variaron en su distribución espacial y vertical, con cuatro especies restringidas al dosel, mientras que las especies con mayor densidad se encontraron entre los 0 y $8 \mathrm{~m}$ (Figura 3). Estas diferencias pueden estar asociadas con: 1) la mayor humedad y menor radiación solar en los estratos bajos (Freiberg, 1994), factores que al evitar la desecación favorecen el crecimiento y desarrollo de grupos como Drymonia, Gasteranthus y Columnea (Weber, 2004); 2) la mayor disponibilidad de sustratos en estratos bajos (Nieder, et al., 2000), que permite su rápida colonización; 3) los mecanismos de dispersión asociados con la polinización por colibríes (Amaya-Márquez, 1996, 1999), y el consumo de frutos por aves (Restrepo, et al., 1999), y 4) la dificultad de detección de las especies del dosel (Smith, 2008). Además, esta segregación vertical permite la coexistencia de diferentes especies de Columnea al presentar estrategias de polinización contrastantes (Stiles,1981; Ackerman, 1986), como son un mayor número de flores en las especies del dosel, una alta sincronía de la floración, un sistema autocompatible y su asociación con colibríes no ermitaños; en comparación con las especies del sotobosque, que presentan una baja intensidad de la floración, tienden a ser polinizadas por colibríes ermitaños y presentan un sistema principalmente autoincompatible (Marín-Gómez, observación personal).

Las plantas de Columnea son polinizadas por colibríes, entre los cuales se encuentran los de pico largo (Phaethornis syrmatophorus, Phaethornis yaruqui y Coeligena wilsoni), que forrajean a lo largo de rutas y que facilitan la polinización cruzada (Marín-Gómez, observación personal), además de ser dispersadas por aves (Restrepo, et al. 1999). Las aves frugívoras pueden determinar la distribución espacial agregada en especies de Rubiaceae y Melastomataceae (Loiselle \& Blake, 1993). Si bien la conducta de forrajeo de aves como las tangaras, que viajan en bandadas mixtas, se ha asociado con la dispersión de Columnea desde el 
bosque a zonas de rastrojo (Amaya-Márquez, 1999), se requiere evidencia que dé soporte a esta hipótesis, dado que aves frugívoras pequeñas como Euphonia xanthogaster, Chlorospingus flavigularis, Tangara rufigularis y Chlorochrysa phoenicotis, que consumen los frutos de Columnea, son especies que exprimen la pulpa del fruto con el pico dejando caer la mayoría de las semillas cerca de la planta, lo cual puede restringir la dispersión a larga distancia (Levey, 1987). Además, si los frutos no son dispersados por aves, se desprenden de la planta y caen cerca de ésta, donde pueden germinar, o en un caso excepcional, como ocurre en C. medicinalis, las semillas pueden germinar en las brácteas extraflorales al descomponerse la pulpa.

\section{Conclusiones}

Los resultados de este estudio indican que la Reserva Natural Río Ñambí alberga la riqueza de Columnea más alta registrada para cualquier localidad hasta el momento, lo que resalta su importancia para la conservación de este grupo de plantas debido a su distribución restringida y su sensibilidad a la deforestación (Kvist, et al., 2004). La mayor densidad se encontró en la zona baja de la reserva, donde predomina una cobertura de bosque intervenido, aunque otras especies (C. longinervosa, C. rubriacuta y C. filifera) son más abundantes en el bosque maduro. Cinco especies fueron epifitas y solo cuatro estuvieron restringidas al dosel, en tanto que las especies restantes fueron hemiepifitas y presentaron una distribución agregada. Este patrón puede estar relacionado con el alto nivel de humedad, el uso de sustratos, las diferencias microclimáticas y las relaciones con sus polinizadores y dispersores. Por lo tanto, las diferencias en la densidad y en la distribución espacial y vertical de las especies de Columnea pueden ser factores importantes que determinan la interacción con los colibríes y la coexistencia de especies con similitudes en cuanto a sus requerimientos de polinización.

\section{Agradecimientos}

Esta investigación se llevó a cabo gracias al apoyo financiero recibido de la beca Nellie D. Sleeth Scholarship (The Gesneriad Society INC), y del programa de incentivos para la investigación Thomas van der Hammen (Jardín Botánico José Celestino Mutis). Agradecemos a la Fundación Ecológica Los Colibríes de Altaquer y a Mauricio Flórez Pai por facilitar las condiciones para la investigación en la reserva, y a Diana, Jairo, Hugo y Guillermo "El Zambo", por su colaboración en la logística de la fase de campo; al Herbario Nacional Colombiano (COL) por permitir la revisión de ejemplares de colección, a la Facultad de Ciencias de la Universidad Nacional de Colombia por la beca otorgada para la realización de los estudios de maestría del primer autor, así como a John L. Clark por sus comentarios al manuscrito, y a James Smith, Laura Clavijo y John L. Clark por sus observaciones taxonómicas en campo. Gary Stiles, Juan Francisco Ornelas y Margarita López revisaron la primera versión del manuscrito. MAM agradece a la Universidad Nacional de Colombia por su apoyo para realizar la investigación.

\section{Conflicto de intereses}

Los autores declaran que no tienen ningún conflicto de intereses.

\section{Bibliografía}

Ackerman, J. D. 1986. Coping with the epiphytic existence: pollination strategies. Selbyana. 9: 52-60.

Amaya-Márquez, M. 1996. Sistemática y polinización del género Columnea (Gesneriaceae) en la Reserva Natural La Planada (Nariño). Tesis de maestría, Universidad Nacional de Colombia, Bogotá.

Amaya-Márquez, M. 1999. Densidad de Columnea (Gesneriaceae) en relación con la edad de un bosque de neblina (La Planada: Nariño, Colombia). Revista de la Academia Colombiana de Ciencias Exactas, Físicas y Naturales. 32: 123-131.

Amaya-Márquez, M., Stiles, F. G., Rangel, O. 2001. Interacción planta-colibrí en Amacayacu (Amazonas, Colombia): una perspectiva palinológica. Caldasia. 23 (1): 301-322.

Arévalo, R. \& Betancur, J. 2006. Vertical distribution of vascular epiphytes in four forest types of the Serranía de Chiribiquete, Colombian Guayana. Selbyana. 27 (2): 175-185.

Barthlott, W., Mutke, J., Rafiqpoor, D., Kier, G., Kreft, H. 2005. Global centers of vascular plant diversity. Nova Acta Leopoldina. 342: 61-83.

Benavides, A. M., Wolf, H. D., Duivenvoorden, J. F. 2006. Recovery and succession of epiphytes in upper Amazonian fallows. Journal of Tropical Ecology. 22: 705-717.

Benzing, D. H. 1990. Vascular epiphytes. General biology and related biota. Cambridge: Cambridge University Press.

Cavelier, J., Jaramillo, M., Solis, D., de León, D. 1997. Water balance and nutrient inputs in bulk precipitation in tropical montane cloud forest in Panama. Journal of Hydrology. 193: 83-96.

Clark, J.L., Neil, D.A., Asanza, M. 2006. Floristic checklist of the Mache-Chindul Mountains of Northwestern Ecuador. Smithsonian Institution Contributions from the United States National Herbarium. 54: 1-180.

Dodson, C.H. \& Gentry, A.H. 1978. Gesneriaceae. In: Flora of the Rio Palenque Science Center. Selbyana. 4: 334-349, 590, 592, 600 .

Elzinga, C. L., Salzer, D. W., Willoughby, J. W. 1998. Measuring and monitoring plant populations. Bureau of Land Management National Business Center. Denver.

Feinsinger P., Tiebout III, H. M., Young, B. E. 1991. Do tropical bird-pollinated plants exhibit density-dependent interactions? Field experiments. Ecology. 72: 1953-1963.

Franco-Rosselli, P, Betancur, J., Fernández-Alonso, J. L. 1997. Diversidad florística en dos bosques subandinos del sur de Colombia. Caldasia. 19: 205-234. 
Freiberg, M. 1994. Phanomorphologie epiphytischer Gesneriaceen in Costa Rica unter besonderer Berucksichtigung des Mikroklimas. Thesis, Universitat Ulm, Germany. 143 p.

Galeano, G., Suárez, S., Balslev, H. 1998. Vascular plant species count in a wet forest in the Chocó area on the Pacific coast of Colombia. Biodiversity and Conservation. 7: 1563-1575.

Gentry, A. H. \& Dodson, C. H. 1987. Diversity and biogeography of neotropical vascular epiphytes. Annals of the Missouri Botanical Garden. 74: 205-233.

González, C. E. \& Jarvis, A. 2002. Plants of Tambito I. Dicotiledonous. A Preliminary List. URL: http://gisweb. ciat.cgiar.org/sig/download/dicotiledonousplantsoftambito. pdf

Gutiérrez-Zamora., A., Carrillo, E., Rojas, S. 2004. Guía Ilustrada de los colibríes de la Reserva Natural Río Nambí. FPAA, FELCA, ECOTONO. Bogotá; Colombia.

Jaramillo, J. L. 2001. Gesneriaceae.. En: Nieder, J. \& W. Barthlott. (Eds.). The flora of the río Guajalito mountain rain forest (Ecuador). p. 144-147. Bonn, Germany.

Jaramillo, J. L. \& Jorgensen, P. M. 1989. Inventario florístico de la Reserva Endesa. En: Jorgensen, P. M. \& C. Ulloa. (Eds.). Estudios botánicos en la Reserva Endesa, Pichincha, Ecuador. p. 1-26. Aarhus Denmark: Botanical Institute, University of Aarhus.

Kelly, D. L., Tanner, E. V., Lughadha, E. M., Kapos, V. 1994. Floristics and biogeography of a rain forest in the Venezuelan Andes. Journal of Biogeography. 21: 421-440.

Köster, N., Kreft, H., Nieder, J., Barthlott, W. 2013. Range size and climatic niche correlate with the vulnerability of epiphytes to human land use in the tropics. Journal of Biogeography. 40: 963-976.

Krebs, C. J. 1999. Ecological Methodology. 2nd ed. Benjamin Cummings Publishers.

Kvist, L. P. \& Skog, L. E. 1993. The genus Columnea (Gesneriaceae) in Ecuador. Allertonia. 6: 327-400.

Kvist, L. P., Skog, L.E., Clark, J. L., Dunn, R. W. 2004. The family Gesneriaceae as example for the biological extinction in Western Ecuador. Lyonia. 6: 127-151.

Levey, D. J. 1987. Seed Size and Fruit-Handling Techniques of Avian Frugivores. The American Naturalist. 129 (4): 471485.

Loiselle B. A. \& Blake, J. G. 1993. Spatial distribution of understory fruit-eating birds and fruiting plants in a Neotropical lowland wet forest. Vegetatio. 108: 177-189.

Mendoza-Cifuentes, H. \& Ramírez-Padilla, B. 2001. Dicotiledóneas de la Planada, Colombia: lista de especies. Biota Colombiana. 2 (1): 59-74.

Möller, M. \& Clark, J. L. 2013. The State of Molecular Studies in the Family Gesneriaceae: A Review. Selbyana. 31 (2): 95-125.

Nadkarni, N. M. \& Matelson, T. J. 1989. Bird use of epiphyte resources in Neotropical trees. The Condor. 91: 891-907.
Nieder, J., Engwald, S., Klawun, M., Barthlott, W. 2000. Spatial Distribution of Vascular Epiphytes (including Hemiepiphytes) in a Lowland Amazonian Rain Forest (Surumoni Crane Plot) of Southern Venezuela. Biotropica. 32 (3): 385-396.

Nieder, J., Prosperi, J., Michaloud, G. 2001. Epiphytes and their contribution to canopy diversity. Plant Ecology, 153: 51-63.

Oksanen, J., Blanchet, F. G., Kindt, R., Legendre, P., Minchin, P. R., O’Hara, R. B., Simpson, G. L., Solymos, P., Henry, M., Stevens, H., Wagner, H. 2013. vegan: Community Ecology Package. R package version 2.0-10. http:// CRAN.R-project.org/package=vegan

Rangel-Ch, J.O. 2004. (Ed.) Colombia Diversidad Biótica IV. El Chocó biogeográfico-Costa Pacífica. Instituto de Ciencias Naturales. Unidad de Monitoreo y Modelaje. Centro para la conservación de la biodiversidad CBC. ANDES. C. Internacional. 997 p. Bogotá.

Restrepo, C., Gómez, N., Heredia, S. 1999. Anthropogenic edges, tree fall gaps, and fruit-frugivore interactions in a Neotropical montane forest. Ecology. 80: 668-685.

Salinas, I. F., Arroyo, T. K., Armesto, J. 2010. Epiphytic growth habits of Chilean Gesneriaceae and the evolution of epiphytes within the tribe Coronanthereae. Annals of the Missouri Botanical Garden. 97 (1): 117-127.

Salaman, P. 2001. The study of an understory avifauna community in an Andean Premontane Pluvial Forest. D. Phil thesis. University of Oxford, U.K

Silverstone-Sopkin, P. A. \& Ramos-Pérez, J. E. 1995. Floristic Exploration and Phytogeography of the Cerro del Torrá, Chocó, Colombia. In: Biodiversity and Conservation of Neotropical Montane Forest. Churchill, S. P; H. Balslev; E. Forero \& J. L. Luteyn. (Eds.). The New York Botanical Garden. New York. 169-186 p.

Smith, J. F. 1994. Systematics of Columnea section Pentadenia and section Stygnanthe (Gesneriaceae). Systematic Botany Monographs. 44: 1-89.

Smith, J. F., Amaya-Márquez., M., Marín-Gómez, O. H., Clark, J. L. 2013. Four New Species of Columnea (Gesneriaceae) with Primary Distributions in Colombia. Journal of the Botanical Research Institute of Texas. 7 (2): 667-679.

Stiles, F. G. 1981.Geographical aspects of bird-flower coevolution, with particular reference to Central America. Annals of the Missouri Botanical Garden. 68: 323-351.

Stuntz, S., Ziegler, C., Simon, U., Zotz, G. 2002. Diversity and structure of the arthropod fauna within three canopy epiphyte species in central Panama. Journal of Tropical Ecology. 18: 161-176.

Weber, A. 2004. Gesneriaceae. In: Kubitzki, K. \& J.W. Kadereit. (Eds.). The Families and Genera of Vascular Plants. Vol. 7. Flowering plants, dicotyledons: Lamiales (except Acanthaceae including Avicenniaceae). p. 63-158. Berlin \& Heidelberg: Springer-Verlag.

Weber, A., Clark, J.L., Möller, M. 2013. A new formal classification of Gesneriaceae. Selbyana. 31: 68-94. 
Wester, S., Mendieta-Leiva, G., Nauheimer, L., Wanek, W., Kreft, H., Zotz, G. 2011. Physiological diversity and biogeography of vascular epiphytes at Río Changuinola, Panama. Flora-Morphology Distribution, Functional Ecology of Plants. 206: 66-79.

Wiehler, H. 1983. A synopsis of the neotropical Gesneriaceae. Selbyana. 6: 1-249.

Zar, J. H. 1999. Biostatistical Analysis. 4th ed. Prentice Hall, Upper Saddle River, New Jersey.
Zotz, G. 2013.The systematic distribution of vascular epiphytes - a critical update. Botanical Journal of the Linnean Society. 171: 453-481.

Zotz, G., Bermejo, P., Dietz, H.1999. The epiphyte vegetation of Annonaglabra on Barro Colorado Island, Panama. Journal of Biogeography. 26: 761-776.

Zotz, G. \& Schultz, S. 2008. The vascular epiphytes of a lowland forest in Panama - species composition and spatial structure. Plant Ecology. 195: 131-141. 